\title{
Socioeconomic differentials in the uptake of medical care in Great Britain
}

\author{
R BALARAJAN, ${ }^{1}$ P YUEN, ${ }^{1}$ AND D MACHIN 12 \\ From the Clinical Epidemiology \& Public Health Research Unit, ${ }^{\prime}$ Robens Institute, University of Surrey, Guildford \\ GU2 5XH; and Medical Statistics and Computing, ${ }^{2}$ University of Southampton, South Academic Block, \\ Southampton General Hospital, Southampton SO9 4XY
}

SUMMARY Socioeconomic differentials in the uptake of medical care in men were investigated using data from the General Household Survey (1974-76 and 1979-80). Hospitalisation, attendance at out patient clinics, and consultation with doctors were higher in local authority tenants than in owner occupiers. Men belonging to the manual socioeconomic groups also used services and consulted doctors more often than those in the non-manual groups. Hospitalisation among men was highest among manual workers living in local authority properties without access to car(s) (OR 1.92). Highest attendance at outpatient clinics (OR 1.60) was seen among local authority tenants in urban areas belonging to manual groups. However, consultation with doctors was highest among urban manual workers living in local authority properties but with access to car(s) (OR 1.72). The implications of these findings for resource allocation are discussed.

\section{Background}

The basis for allocating health resources in England is the population of the region, modified by age-sex structure and the mortality experience. ${ }^{1}$ The latter is known to differ among the various socioeconomic groups. $^{2-4}$ At present no consideration is given in the process of resource allocation for the needs arising from the different socioeconomic distribution of a defined population. We have in this analysis used readily available information from the General Household Survey to study the socioeconomic differentials in the uptake of medical care with a view to their possible application in allocating resources for health care in Great Britain.

\section{Materials and methods}

The General Household Survey (GHS) ${ }^{5}$ is an annual survey based on a sample of the population resident in private households in Great Britain, conducted by the Office of Population Censuses and Surveys (OPCS). The surveys of $1974,1975,1976,1979$, and 1980 were used for this analysis. The choice of years was dictated by the questions asked and the level of data released in the respective years, the latest year for which data were available at the time of the analysis being 1980 . The GHS obtains its sample households through a two- stage rotating design with electoral wards as the primary sampling unit. Information was available on age, sex, socioeconomic group (SEG), housing tenure, access to car(s), and area of residence. A measure of the uptake of health care was also obtained at the time of the interview which covered consultation with a doctor in the previous two weeks and attendance at outpatient or accident and emergency department of a hospital over a reference period of three months. It was not possible to separate the attendance at outpatient and accident and emergency departments in the analysis as they were treated together in the survey. Information on inpatient attendance was available only for the years 1974-76 restricting analyses on this outcome variable to this period. Information for 1974-76 was also incomplete for area of residence and as we were interested in the urban/ rural gradient, the analyses relating to consultation with a doctor and attendance at outpatient departments relate to the later years of 1979-80. In view of the fact that many of the social variables, such as tenure, socioeconomic groups, access to car(s), are determined by the status of the head of household, we have restricted this analysis to men. The elderly were not considered in this study for two reasons. Preliminary investigations demonstrated the importance of other social variables in explaining both their health status and uptake of care, and further the 
GHS contains a particularly select group of elderly people with especially good health, ${ }^{3}$ as it excludes the institutional populations.

Multiple logistic regression analysis was employed to model the probability of both "consultation with a doctor" and "attendance at an outpatient or accident and emergency department" using the following variables for the years 1979 and 1980; socioeconomic group (non-manual, manual); housing tenure (owneroccupier, local authority tenant); access to car(s); area of residence (urban, rural). Men living in their own house in a rural area and engaged in a non-manual occupation with access to car(s) were identified as the baseline group for comparison $(\mathrm{OR}=1)$ in the analysis.

The analysis was repeated for inpatient attendance for the years 1974-76 using the variables available for these years (age, socioeconomic group, housing tenure, and access to cars). The computer package GLIM $^{6}$ was used to obtain, by means of a simultaneous testing procedure, ${ }^{78}$ a "best" fitted model that incorporated those main effects and interactions which significantly reduced the deviance. Maximum likelihood estimates of the odds ratios standardised for age were obtained, and the corresponding $95 \%$ confidence intervals were calculated.

\section{Results}

The distributions of the study population by the different social variables are shown separately for the two study periods in the table.

CONSULTATION WITH A DOCTOR

The main effects model, which included all the

Male population in the study by age, housing tenure, access to car and socioeconomic group (General Household Survey, 1974-76, 79-80)

\begin{tabular}{llrr}
\hline & Access to car & Owner occupier & $\begin{array}{l}\text { Local authority } \\
\text { tenant }\end{array}$ \\
\hline 1974-76 & Yes & 6042 & 1094 \\
Non-manual & No & 581 & 510 \\
Manual & Yes & 5022 & 3666 \\
& No & 1371 & 3488 \\
$1979-80$ & & & \\
Rural area & & 1414 & 135 \\
Non-manual & Yes & 37 & 34 \\
& No & 1020 & 546 \\
Manual & Yes & 102 & 207 \\
& No & & \\
Urban area & & 3294 & 325 \\
Non-manual & Yes & 3050 & 1906 \\
Manual & Yes & 798 & 1922 \\
& No & & \\
\hline
\end{tabular}

variables, was chosen since there were no significant interaction terms. Consultation with a doctor was higher in men who were manual workers, those who resided in urban areas, those who were local authority tenants, and those who had access to car(s). The latter two variables reached formal statistical significance with $\mathrm{OR}=1 \cdot 17, \mathrm{p}=0.01$, and $\mathrm{OR}=1 / 0.81=1.23$, $p<0.001$ respectively (fig 1 ). A main effects model including no interaction terms implies that the effects are multiplicative. Thus, for example, the estimated odds ratio, for consultation among urban manual workers who were local authority tenants but had access to a car(s) was $1.10 \times 1.08 \times 1.17 \times 1.23=$ $1 \cdot 71$.

ATTENDANCE AT OUTPATIENT OR ACCIDENT/EMERGENCY DEPARTMENT

Estimated odds ratios for attending an outpatient or accident and emergency department are shown in figure 2. Attendance was significantly higher among men who were manual workers (OR $1 \cdot 12, p=0.04)$, those who were local authority tenants $(\mathrm{OR} 1 \cdot 21, \mathrm{p}=$

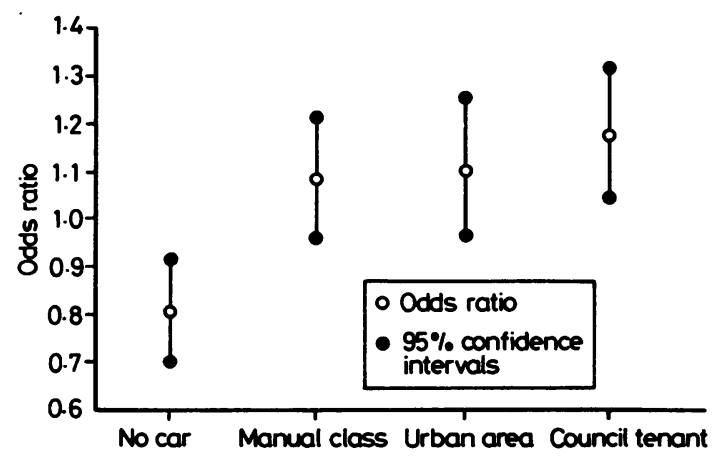

Fig 1 Estimated odds ratios (males) for consultation with a doctor: General Household Survey 1979-80.

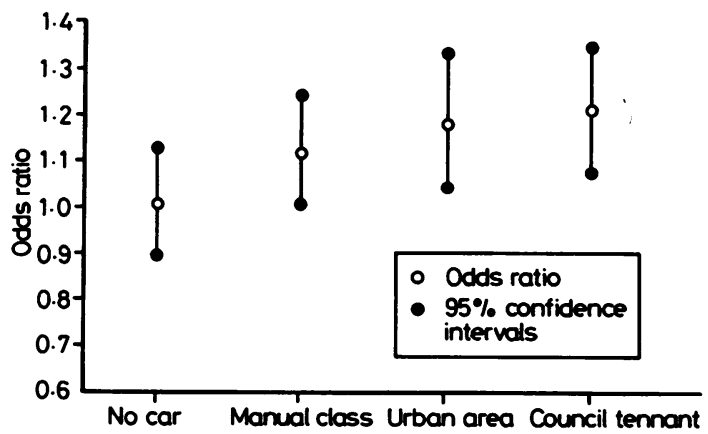

Fig 2 Estimated odds ratios (males) for attending outpatient department. GHS 1979-80. 
0.001 ), and those who lived in urban areas (OR 1.18, $p=0.006$ ). No difference in attendance at outpatient departments was seen between those with and without access to car(s). Those who attended outpatient departments most were male urban manual workers who were local authority tenants $(\mathrm{OR}=1 \cdot 60)$.

\section{ATTENDANCE AS INPATIENT}

Inpatient information available for the years 1974-76 did not include area of residence in sufficient detail for modelling. Local authority tenants were hospitalised significantly more often than owner occupiers (OR $1 \cdot 33, p=0.03$ ) (fig 3). Men who were manual workers were also admitted to hospitals more often than non-manual workers. The same was true for those without access to car(s). The highest hospitalisation was among manual workers who were local authority tenants with no access to car(s) $(O R=1.92)$.

\section{Discussion}

The GHS collects data on a range of social variables providing the opportunity to link these with the information collected on the health status of individuals as well as their health service utilisation. As it is not a dedicated health interview survey some limitations do exist in the use of the data. Furthermore, external validation is not carried out with either hospital or general practitioner records for individuals. However, it remains a unique source of data covering aspects of morbidity and social factors on a large representative population sample in the country.

In our study, men in the manual socioeconomic groups not only consulted a doctor more often but also utilised both outpatient and inpatient facilities more often than those in the non-manual groups. Housing tenure, on the other hand, discriminated most for the uptake of medical care. Men who were

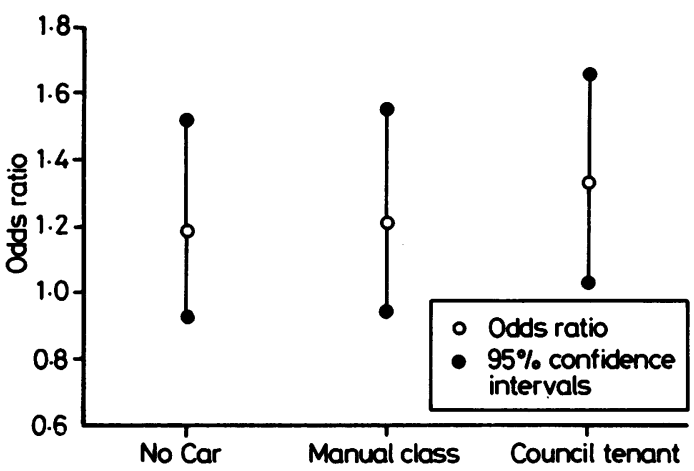

Fig 3 Estimated odds ratios (males) for attending as inpatient. GHS 1974-6. local authority tenants consulted doctors and attended hospitals more often than owner occupiers. Attendance at outpatient clinics and consultation with doctors were more common among urban residents. Lack of access to a car(s) appeared to act as surrogate for social means for inpatient attendance. In contrast, those with access to a car(s) were more likely to consult a doctor. This apparent reversal of what might be anticipated from trends in the other socioeconomic gradients observed may reflect the likely importance of ready access to a means of transport on primary care consultations rates. Access to car(s) appears to have little effect on attendance at outpatient and emergency departments.

All socioeconomic factors examined influenced to varying degrees the uptake of medical care. Thus populations with different socioeconomic distributions will have differential demands for health care. However, these differentials reflect only the current patterns of uptake of medical care, that is, met demand, and could very well be underestimates of the true differences that exist between the different social profiles examined in this study. Perceptions of health needs and thresholds for seeking care are known to vary. 910 Nevertheless, as the gradients are weighted towards the deprived, there are no reasons why these could not be taken as the minimum differentials for need among the social profiles studied. It is important to recognise that these differentials appear independent and multiplicative. For example, the differentials between non-manual workers and manual workers for hospitalisation was $21 \%$, which increased to $61 \%$ if the manual worker was also a local authority tenant and to $92 \%$ if he also did not have a car.

These findings based on available information from the GHS strongly indicate the need for socioeconomic differentials between geographical areas to be considered in resource allocation. There are other social profiles that may merit further examination, such as the distribution of single parent families, ethnic groups, unemployed, and the elderly.

Specially designed studies relating population characteristics to the various health service activities by diagnostic entities could provide more sensitive weightings for consideration in the resource allocation process.

We thank Dr PO Goldblatt, Social Statistics Research Unit, City University; Ms S Arber, Department of Sociology, University of Surrey; Dr JSA Ashley, Department of Community Health, London School of Hygiene and Tropical Medicine, for their helpful comments; the ESRC Data Archive, University of Essex, the Department of Sociology, University of 
Surrey, and the Office of Population Censuses and Surveys for making available the General Household Survey data. We acknowledge in particular the support received from South West Thames Regional Health Authority for this study.

\section{References}

${ }^{1}$ Department of Health and Social Security. Sharing resources for health in England. Report of the Resource Allocation Working Party London: HMSO, 1976

2 Office of Population Censuses and Surveys. Occupational mortality decennial supplement 1970-72. London: HMSO, 1978.
${ }^{3}$ Fox AJ, Goldblatt PO. Socio-demographic mortality differentials from the OPCS Longitudinal Study 1971-75 Series LS No 1. London: HMSO, 1980.

${ }^{4}$ Office of Population Censuses and Surveys. Occupational mortality decennial supplement: 1979-80, 82-83 London: HMSO, 1986.

${ }^{5}$ Office of Population Censuses and Surveys. General Household Survey Introductory Report. London: HMSO, 1973

${ }^{6}$ Baker RJ, Nelder JA. GLIM System (Release 3) Oxford: Numerical Algorithm Group, 1981.

${ }^{7}$ Aitkin M. A simultaneous testing procedure for contingency table models. Appl Stat 1979 28: 233-42

${ }^{8}$ Aitkin M. A note on the selection of log-linear models. Biometrics 1980 36: 173-8

${ }^{9}$ Dunnell K, Cartwright A. Medicine takers, prescribers and hoarders. London: Routledge \& Kegan Paul.

${ }^{10}$ Cameron A, Hinton J. Delay in seeking treatment for mammary tumours. Cancer 1968 21: $1121-6$

Accepted for publication May 1987 